\section{UJMM

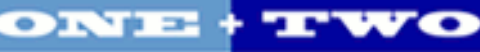

Volume 9 | 2018 Fall 2018

\section{Undergraduate Journal of Mathematical}

Modeling: One + Two

2018

\title{
Elapsed Time of Vehicle Acceleration
}

Jensen McTighe

University of South Florida

Advisors:

Arcadii Grinshpan, Mathematics and Statistics

Don Dekker, Mechanical Engineering

Problem Suggested By: Don Dekker

Follow this and additional works at: https://digitalcommons.usf.edu/ujmm

Part of the Mathematics Commons

UJMM is an open access journal, free to authors and readers, and relies on your support:

Donate Now

\section{Recommended Citation}

McTighe, Jensen (2018) "Elapsed Time of Vehicle Acceleration," Undergraduate Journal of Mathematical Modeling: One + Two: Vol. 9: Iss. 1, Article 5.

DOI: https://doi.org/10.5038/2326-3652.9.1.4898

Available at: https://digitalcommons.usf.edu/ujmm/vol9/iss1/5 


\title{
Elapsed Time of Vehicle Acceleration
}

\begin{abstract}
Newton's Second Law states that force is equal to the mass of an object multiplied by its acceleration. More specifically, force is the mass times the instantaneous change in velocity over time of an object. By rearranging this equation, it can be determined that the time elapsed of the acceleration of an object is equal to the integral of the inverse value of the force relative to change in velocity (dv). In the context of real world application, this method can be used to calculate the time taken for a vehicle to accelerate from its minimum to maximum speed, given the values of torque output relative to engine rpms, transmission specifications, vehicle weight, and tire size. To demonstrate the viability of this method, the elapsed time of acceleration is calculated for a 2017 Ford GT with a torque-rpm curve containing 58 values. It is found that the resulting values are realistic when neglecting forces of friction and air resistance. The results obtained would be analogous to the values obtained through experimentation on a dynamometer, which allows the vehicle to be tested while stationary.
\end{abstract}

\section{Keywords}

vehicle's engine, force, speed, transmission, tire radius, torque-rpm curve, Ford GT, Riemann sum, approximate integration

\section{Creative Commons License}

\section{(c) $($ ) $(9)$}

This work is licensed under a Creative Commons Attribution-Noncommercial-Share Alike 4.0 License. 


\section{Problem STATEMENT}

The purpose of this paper is to calculate the amount of time it takes for a specific vehicle to accelerate to its maximum speed using information obtained from the engine's torque-rpm curve and the specifications of the vehicle.

\section{Motivation}

The ability to calculate the time necessary for a vehicle to go from idle to maximum speed exemplifies the key relationship between engineering, physics, and calculus. From the perspective of the automotive industry, this problem is important because it shows the effects of a combination of a vehicle's engine, transmission, and tire radius on the performance at maximum output. By collecting data on a variety of engines and transmissions, this can then be used to choose the engine and transmission gear ratios best fit for the intended purpose of the vehicle.

\section{MATHEMATICAL DESCRIPTION AND SOLUTION APPROACH}

To calculate the elapsed time of the vehicle acceleration, the specific points of the torquerpm, differential ratio, gear ratio, vehicle weight, and tire radius must be obtained. First, the force at the rear wheels and vehicle speed must be calculated at each point on the torque-rpm curve using equations (1) and (2):

$$
\text { Force }_{R W}(l b f)=\frac{\text { Torque }_{\text {engine }}(\text { lbft }) \times \text { Differential Ratio } \times(\text { Ratio for Specific Gear })}{\text { Tire Radius }(f t)},
$$




$$
\operatorname{Velocity}(f t / s)=\frac{r p m_{\text {engine }}\left(\frac{\text { rev }}{\min }\right) \times \text { Tire Radius }(f t) \times 2 \pi\left(\frac{r a d}{r e v}\right)}{\text { Differential Ratio } \times(\text { Ratio for Specific Gear }) \times 60 \frac{\mathrm{s}}{\mathrm{min}}} .
$$

The force is calculated by multiplying the torque from the engine and the total ratio of the output of the wheels from the engine and dividing by the tire radius which cancels out the unit of feet and yields the force output at the wheels. The velocity is then calculated by multiplying the rpm by the radius of the tire and $2 \pi$. This is done to convert the value from revolutions per minute to radians per minute. Finally, this value is divided by the differential ratio times the ratio of the specific gear times 60 seconds per minute. The calculations result in the velocity of the vehicle over ground in feet per second.

Once the force output and velocity are calculated for each rpm listed, the force inverse must be calculated. Because the force is expressed in pounds force, the equation is simply 1 divided by the force multiplied by the weight over the acceleration due to gravity, $g_{c}$. Once the force inverse (3) is calculated for each point, a graph must be generated of the force inverse relative to velocity for each value of rpm:

$$
\text { Force Inverse }=\frac{m(l b)}{g_{c} \frac{f t}{s^{2}}} \times \frac{1}{\text { Force }_{R W}} \text {. }
$$

A solution to the problem is reached on the basis that force is equal to the mass of the object times the acceleration, treated as the derivative of the velocity:

$$
\operatorname{Force}(\operatorname{lbf})=\frac{m}{g_{c}} \times \frac{d V}{d t}
$$

By rearranging equation (4) and integrating it we obtain formula (5):

$$
\text { Elapsed Time }=\int d t=\int \frac{m}{g_{c}} \times \frac{1}{\text { Force }} \times d V
$$


Taking the integral of the graph of the force inverse relative to velocity will result in the elapsed time of the acceleration of the vehicle. Because the curve obtained is not defined by a specific function, a method of estimation must be used to determine the value of the integral. Based on the curve, a right-Riemann sum will yield an overestimate while a left-Riemann sum will yield an underestimate since the overall slope is positive (see Appendix I and [1, Section 6.5]).

\section{DISCUSSION}

The vehicle selected for this paper is a 2017 Ford GT. The GT is a high-performance car with a 3.5L V6 with a maximum of $647 \mathrm{hp}$ and a 7 -speed transmission. The total weight of the vehicle is $3,170 \mathrm{lb}$ and has a wheel radius of about $1.667 \mathrm{ft}$. A torque-rpm/horsepower curve containing 58 data points is obtained from Automobile Catalog and used as the basis of all calculations (see $[2,3]$ and Appendix II). Below is the torque-rpm and horsepower curve:

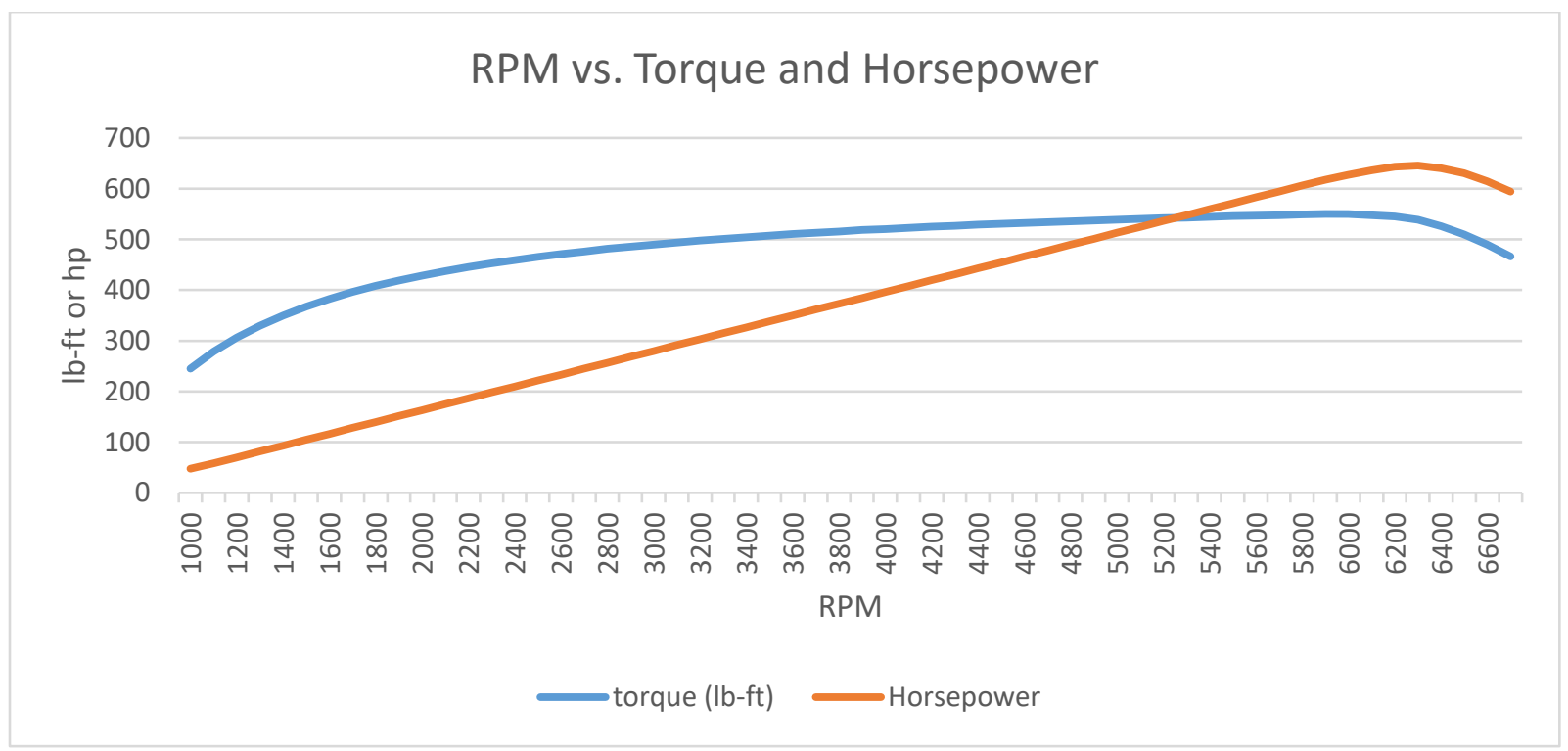

Figure 1 
The force output at the rearwheels and vehicle speed are then calculated relative to rpms and set on a graph of force relative to speed. For a better visual understanding, the calculations are made for each gear and expressed on the graph for each gear.
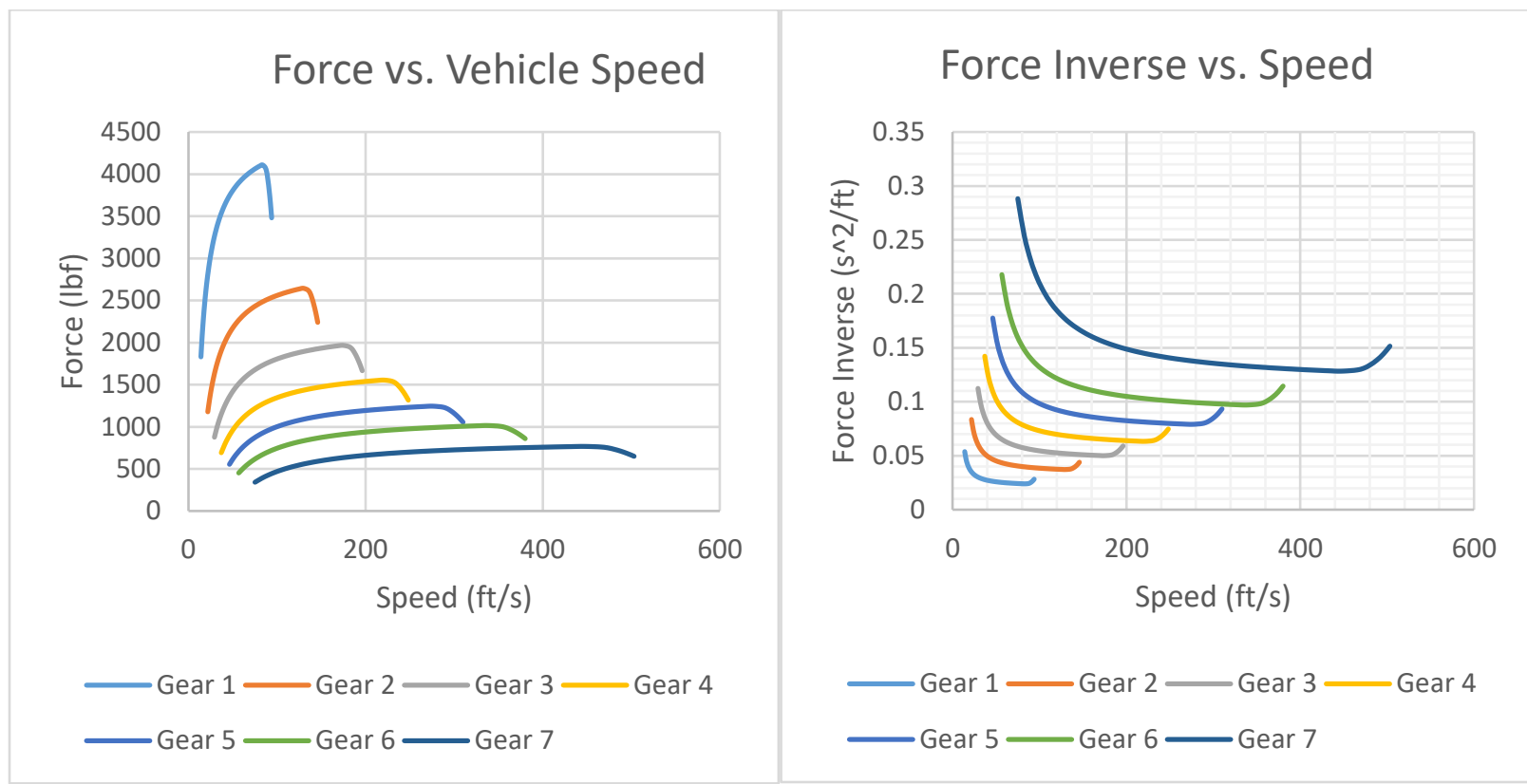

Figure 2 (left) and Figure 3 (right) 
A graph of the force inverse relative to speed is also produced. Finally, the left and right Riemann sums are taken using all of the data points obtained. The time elapsed calculated is 21.37s for a right Riemann sum and 19.01s for a left Riemann sum. It should be noted that when taking the area under the curve, since there are 7 different curves which have overlapping areas, the sum is calculated by taking the points on the curves in which there are not any other values of the other curves beneath. An additional graph of only the points used for the estimated area under the curve can better illustrate this.

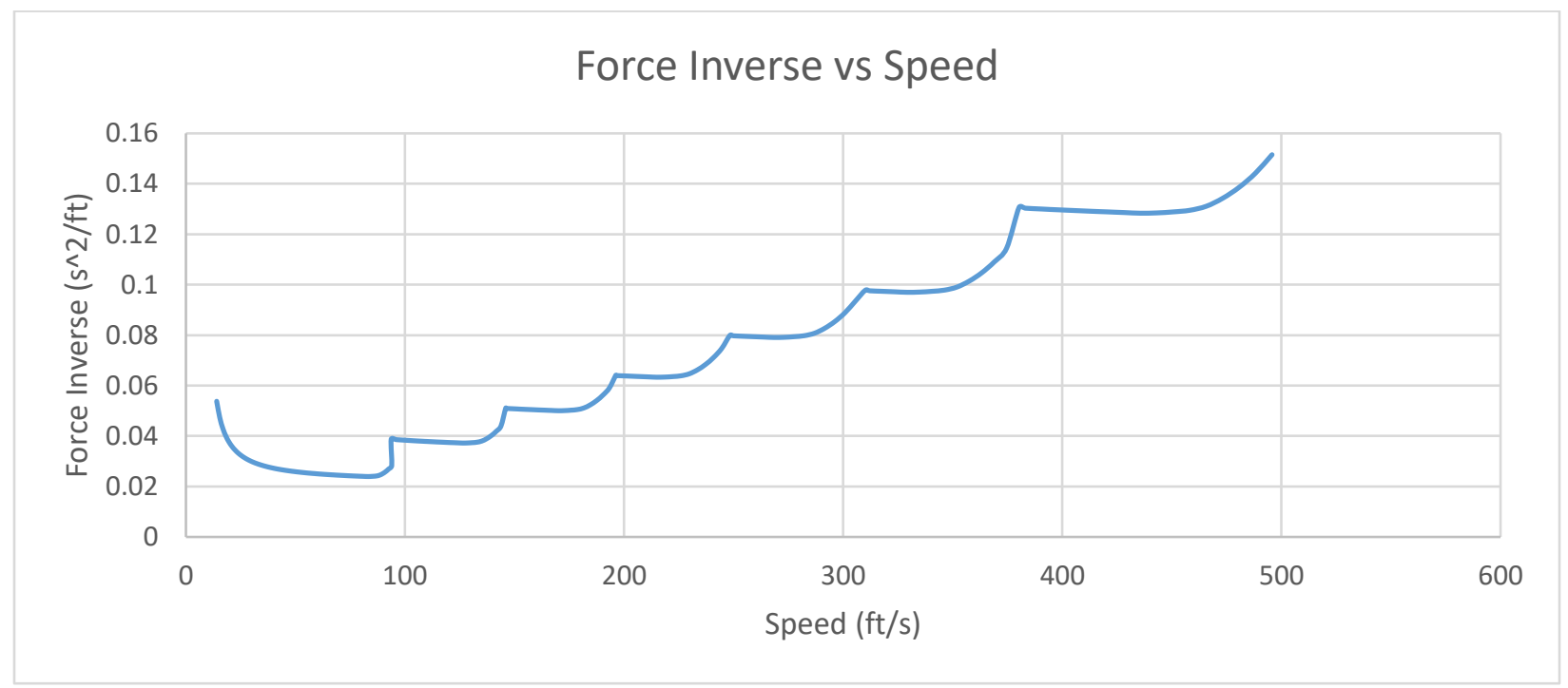

Figure 4 
The results obtained are rather realistic taken into consideration internal and external factors such as friction and air resistance. The results demonstrate the pattern in which a vehicle accelerates based on its engine specifications and transmission gear ratios as well as the difference in how each gear ratio affects the acceleration of the vehicle. As portrayed in Figure 2 and Figure 3, the curve for each gear is very similar. However, each successive gear has a lower overall force output and can reach a higher magnitude of velocity. This demonstrates how each transmission gear ratio is selected to be used within a specific range of velocity to achieve a very high maximum speed while accelerating as much as possible.

It should be noted that the values in Figure 4 are not plotted starting from 0 for velocity. This is because under the minimum speed, the clutch on the vehicle is not fully engaged. Therefore, a portion of the torque produced from the engine is not being delivered to the rear wheels. The maximum speed of the vehicle is also unrealistically high in magnitude because the calculations negate air resistance, external forces of friction (friction between the tires and the road), and internal forces of friction (friction between moving parts within the vehicle).

\section{CONCLUSIONS AND RECOMMENDATIONS}

The data concludes that the method of calculating the elapsed time of the acceleration of a vehicle by isolating the change in time $(d t)$ in Newton's Second Law is viable. In a situation where a vehicle's performance is measured while it is stationary, the results of the time elapsed would most likely be very similar to the true values recorded experimentally with a dynamometer. This method could be valuable for optimizing the transmission gear ratio to 
provide maximum vehicle acceleration while being able to reach the highest speed possible, specifically in vehicles built for high performance.

When using this method, in order to reduce a potential error, it would be beneficial to use a form of integral estimation that is more accurate than a left or right Riemann sum. Ideally, a better form of estimation would be to use Simpson's Rule. This form of estimation is not used because the number of values obtained would not follow the formula needed. In such a case, Simpson's Rule could be combined with a trapezoidal Riemann sum. Estimating in this matter would decrease the error in the resulting elapsed time. Only using a trapezoidal Riemann sum would also reduce the error but this is not the most accurate method (see Appendix I and $[1$, Section 6.5]). 
NOMENCLATURE

\begin{tabular}{|c|c|c|}
\hline Symbol & Value & Units \\
\hline $\mathrm{lbf}$ & Pounds Force & $\frac{l b \times f t}{s^{2}}$ \\
\hline$g_{c}$ & Acceleration Due to Gravity & $32.2 \frac{f t}{s^{2}}$ \\
\hline $\mathrm{rpm}$ & Revolutions per Minute & revs \\
\hline$m$ & Weight (Pounds) & $l b$ \\
\hline
\end{tabular}

\section{REFERENCES}

1. James Stewart, Essential calculus: Early Transcendentals, 2nd Edition. Belmont, CA: Brooks/Cole, Cengage Learning, 2013.

2. Automobile Catalogue. 2017 Ford GT engine Horsepower / Torque Curve. http://www.automobile-catalog.com/bigcurve/2017/2563970/ford_gt.html .

3. Automobile Catalogue. Ford GT (d-cl. 7) (2017) full detailed specifications listing and photo gallery. http://www.automobile-catalog.com/auta_details1.php . 


\section{APPENDIX I}

\section{[1, Section 6.5]}

Any definite integral $\int_{a}^{b} f(x) d x$ can be approximated by the corresponding Riemann sums. If we divide the interval $[a, b]$ into $n$ subintervals of equal length $\Delta x=(b-a) / n$, then we have:

$$
\int_{a}^{b} f(x) d x \approx \sum_{k=1}^{n} f\left(c_{k}\right) \Delta x
$$

where $c_{k}$ is any point in the $k$-th subinterval $\left[x_{k-1}, x_{k}\right]$. If $c_{k}$ is chosen to be the left end-point of the interval, then $c_{k}=x_{k-1}$ and we have the left endpoint approximation:

$$
\int_{a}^{b} f(x) d x \approx \sum_{k=1}^{n} f\left(x_{k-1}\right) \Delta x
$$

If we choose $c_{k}$ to be the right end-point, then $c_{k}=x_{k}$ and we have the right endpoint approximation:

$$
\int_{a}^{b} f(x) d x \approx \sum_{k=1}^{n} f\left(x_{k}\right) \Delta x
$$

The Trapezoidal approximation results from averaging the left and right endpoint approximations:

$$
\int_{a}^{b} f(x) d x \approx \frac{\Delta x}{2} \times \sum_{k=1}^{n}\left[f\left(x_{k-1}\right)+f\left(x_{k}\right)\right] .
$$

Simpson's rule for approximate integration:

$$
\begin{gathered}
\int_{a}^{b} f(x) d x \\
\approx \frac{\Delta x}{3} \times \sum_{k=1}^{n}\left[f\left(x_{0}\right)+4 f\left(x_{1}\right)+2 f\left(x_{2}\right)+4 f\left(x_{3}\right)+\cdots+2 f\left(x_{n-2}\right)+4 f\left(x_{n-1}\right)+f\left(x_{n}\right)\right]
\end{gathered}
$$

results from using parabolas to approximate a curve $y=f(x)$. 


\section{APPENDIX II}

Table 1. (Engine rpm vs. Torque and Horsepower)

\begin{tabular}{|c|c|c|}
\hline rpm & to rque $(\mathrm{lb}-\mathrm{ft})$ & Horsepower \\
\hline 1000 & 245 & 47.6 \\
\hline 1100 & 278.4 & 58.3 \\
\hline 1200 & 306.2 & 69.9 \\
\hline 1300 & 329.8 & 81.6 \\
\hline 1400 & 349.9 & 93.2 \\
\hline 1500 & 367.5 & 104.9 \\
\hline 1600 & 382.7 & 116.5 \\
\hline 1700 & 396.3 & 128.2 \\
\hline 1800 & 408.3 & 139.8 \\
\hline 1900 & 419 & 151.5 \\
\hline 2000 & 428.7 & 163.1 \\
\hline 2100 & 437.5 & 174.8 \\
\hline 2200 & 445.4 & 186.5 \\
\hline 2300 & 452.7 & 198.1 \\
\hline 2400 & 459.3 & 209.8 \\
\hline 2500 & 465.4 & 221.4 \\
\hline 2600 & 471.1 & 233.1 \\
\hline 2700 & 476.3 & 244.7 \\
\hline 2800 & 481.2 & 256.4 \\
\hline 2900 & 485.7 & 268 \\
\hline 3000 & 490 & 279.7 \\
\hline 3100 & 493.9 & 291.3 \\
\hline 3200 & 497.6 & 303 \\
\hline 3300 & 501.1 & 314.7 \\
\hline 3400 & 504.4 & 326.3 \\
\hline 3500 & 507.4 & 338 \\
\hline 3600 & 510.3 & 349.6 \\
\hline 3700 & 513.1 & 361.3 \\
\hline 3800 & 515.7 & 372.9 \\
\hline 3900 & 518.2 & 384.6 \\
\hline 4000 & 520.6 & 396.2 \\
\hline 4100 & 522.8 & 407.9 \\
\hline 4200 & 524.9 & 419.5 \\
\hline 4300 & 527 & 431.2 \\
\hline 4400 & 528.9 & 442.8 \\
\hline 4500 & 530.8 & 454.5 \\
\hline 4600 & 532.5 & 466.1 \\
\hline 4700 & 534.2 & 477.8 \\
\hline 4800 & 535.8 & 489.4 \\
\hline 4900 & 537.5 & 501.1 \\
\hline 5000 & 538.9 & 512.8 \\
\hline 5100 & 540.4 & 524.4 \\
\hline 5200 & 541.7 & 536 \\
\hline 5300 & 543.1 & 547.7 \\
\hline 5400 & 544.4 & 559.4 \\
\hline 5500 & 545.6 & 571.1 \\
\hline 5600 & 546.8 & 582.7 \\
\hline 5700 & 547.9 & 594.3 \\
\hline 5800 & 549 & 606 \\
\hline 5900 & 550.1 & 617.6 \\
\hline 6000 & 549.6 & 627.5 \\
\hline 6100 & 547.9 & 636 \\
\hline 6200 & 545.2 & 643.2 \\
\hline 6300 & 538.6 & 645.6 \\
\hline 6400 & 525.9 & 640.4 \\
\hline 6500 & 509.4 & 630.1 \\
\hline 6600 & 489.4 & 614.6 \\
\hline 6700 & 465.9 & 593.9 \\
\hline
\end{tabular}


M cTighe: Elapsed Time of V ehicle Acceleration

Table 2. (Torque (lb-ft) vs. Force (lbf) per gear)

\begin{tabular}{|c|c|c|c|c|c|c|c|}
\hline torque (lb-ft) & 1 & 2 & 3 & 4 & 5 & 6 & 7 \\
\hline 245 & 1830.284 & 1177.804 & 876.0794 & 692.3506 & 554.4193 & 452.0484 & 341.5955 \\
\hline 278.4 & 2079.8 & 1338.37 & 995.5123 & 786.7364 & 630.0013 & 513.6746 & 388.1641 \\
\hline 306.2 & 2287.482 & 1472.015 & 1094.921 & 865.297 & 692.9109 & 564.9682 & 426.9247 \\
\hline 329.8 & 2463.786 & 1585.469 & 1179.31 & 931.9887 & 746.3162 & 608.5125 & 459.8294 \\
\hline 349.9 & 2613.944 & 1682.097 & 1251.184 & 988.7897 & 791.8012 & 645.5989 & 487.8542 \\
\hline 367.5 & 2745.426 & 1766.706 & 1314.119 & 1038.526 & 831.6289 & 678.0726 & 512.3933 \\
\hline 382.7 & 2858.978 & 1839.778 & 1368.472 & 1081.48 & 866.0255 & 706.118 & 533.5862 \\
\hline 396.3 & 2960.578 & 1905.158 & 1417.103 & 1119.912 & 896.8015 & 731.2113 & 552.5482 \\
\hline 408.3 & 3050.224 & 1962.847 & 1460.013 & 1153.823 & 923.9567 & 753.3524 & 569.2794 \\
\hline 419 & 3130.159 & 2014.286 & 1498.275 & 1184.061 & 948.1701 & 773.095 & 584.1981 \\
\hline 428.7 & 3202.624 & 2060.917 & 1532.96 & 1211.472 & 970.1206 & 790.9924 & 597.7225 \\
\hline 437.5 & 3268.364 & 2103.222 & 1564.428 & 1236.34 & 990.0344 & 807.2292 & 609.9921 \\
\hline 445.4 & 3327.382 & 2141.2 & 1592.677 & 1258.665 & 1007.912 & 821.8055 & 621.0068 \\
\hline 452.7 & 3381.917 & 2176.294 & 1618.78 & 1279.294 & 1024.431 & 835.2747 & 631.1849 \\
\hline 459.3 & 3431.222 & 2208.022 & 1642.381 & 1297.945 & 1039.366 & 847.4523 & 640.3871 \\
\hline 465.4 & 3476.793 & 2237.347 & 1664.193 & 1315.184 & 1053.17 & 858.7074 & 648.8921 \\
\hline 471.1 & 3519.375 & 2264.749 & 1684.576 & 1331.291 & 1066.069 & 869.2244 & 656.8394 \\
\hline 476.3 & 3558.222 & 2289.748 & 1703.17 & 1345.986 & 1077.836 & 878.8189 & 664.0896 \\
\hline 481.2 & 3594.827 & 2313.304 & 1720.692 & 1359.833 & 1088.925 & 887.8599 & 670.9215 \\
\hline 485.7 & 3628.445 & 2334.937 & 1736.783 & 1372.55 & 1099.108 & 896.1628 & 677.1957 \\
\hline 490 & 3660.568 & 2355.608 & 1752.159 & 1384.701 & 1108.839 & 904.0967 & 683.1911 \\
\hline 493.9 & 3689.703 & 2374.357 & 1766.105 & 1395.722 & 1117.664 & 911.2926 & 688.6287 \\
\hline 497.6 & 3717.344 & 2392.144 & 1779.335 & 1406.178 & 1126.037 & 918.1195 & 693.7875 \\
\hline 501.1 & 3743.491 & 2408.97 & 1791.851 & 1416.069 & 1133.957 & 924.5773 & 698.6675 \\
\hline 504.4 & 3768.144 & 2424.834 & 1803.651 & 1425.394 & 1141.425 & 930.6661 & 703.2686 \\
\hline 507.4 & 3790.556 & 2439.257 & 1814.378 & 1433.872 & 1148.214 & 936.2014 & 707.4514 \\
\hline 510.3 & 3812.22 & 2453.198 & 1824.748 & 1442.067 & 1154.776 & 941.5522 & 711.4947 \\
\hline 513.1 & 3833.138 & 2466.659 & 1834.761 & 1449.98 & 1161.112 & 946.7184 & 715.3987 \\
\hline 515.7 & 3852.561 & 2479.158 & 1844.058 & 1457.327 & 1166.996 & 951.5157 & 719.0238 \\
\hline 518.2 & 3871.238 & 2491.176 & 1852.997 & 1464.392 & 1172.653 & 956.1284 & 722.5094 \\
\hline 520.6 & 3889.167 & 2502.714 & 1861.579 & 1471.174 & 1178.084 & 960.5567 & 725.8557 \\
\hline 522.8 & 3905.602 & 2513.29 & 1869.446 & 1477.391 & 1183.063 & 964.6159 & 728.9231 \\
\hline 524.9 & 3921.29 & 2523.385 & 1876.955 & 1483.326 & 1187.815 & 968.4906 & 731.851 \\
\hline 527 & 3936.978 & 2533.481 & 1884.465 & 1489.26 & 1192.567 & 972.3653 & 734.779 \\
\hline 528.9 & 3951.172 & 2542.615 & 1891.259 & 1494.63 & 1196.867 & 975.8709 & 737.4281 \\
\hline 530.8 & 3965.366 & 2551.749 & 1898.053 & 1499.999 & 1201.166 & 979.3766 & 740.0772 \\
\hline 532.5 & 3978.066 & 2559.921 & 1904.132 & 1504.803 & 1205.013 & 982.5133 & 742.4475 \\
\hline 534.2 & 3990.766 & 2568.094 & 1910.211 & 1509.607 & 1208.86 & 985.65 & 744.8177 \\
\hline 535.8 & 4002.719 & 2575.786 & 1915.932 & 1514.128 & 1212.481 & 988.6021 & 747.0486 \\
\hline 537.5 & 4015.419 & 2583.958 & 1922.011 & 1518.932 & 1216.328 & 991.7388 & 749.4188 \\
\hline 538.9 & 4025.878 & 2590.689 & 1927.017 & 1522.889 & 1219.496 & 994.3219 & 751.3708 \\
\hline 540.4 & 4037.084 & 2597.9 & 1932.381 & 1527.128 & 1222.891 & 997.0895 & 753.4622 \\
\hline 541.7 & 4046.795 & 2604.149 & 1937.03 & 1530.801 & 1225.832 & 999.4882 & 755.2747 \\
\hline 543.1 & 4057.254 & 2610.879 & 1942.036 & 1534.758 & 1229 & 1002.071 & 757.2267 \\
\hline 544.4 & 4066.966 & 2617.129 & 1946.684 & 1538.431 & 1231.942 & 1004.47 & 759.0393 \\
\hline 545.6 & 4075.93 & 2622.898 & 1950.975 & 1541.822 & 1234.658 & 1006.684 & 760.7124 \\
\hline 546.8 & 4084.895 & 2628.667 & 1955.266 & 1545.214 & 1237.373 & 1008.898 & 762.3855 \\
\hline 547.9 & 4093.113 & 2633.955 & 1959.2 & 1548.322 & 1239.863 & 1010.928 & 763.9192 \\
\hline 549 & 4101.33 & 2639.243 & 1963.133 & 1551.431 & 1242.352 & 1012.957 & 765.4529 \\
\hline 550.1 & 4109.548 & 2644.531 & 1967.067 & 1554.539 & 1244.841 & 1014.987 & 766.9866 \\
\hline 549.6 & 4105.813 & 2642.127 & 1965.279 & 1553.126 & 1243.71 & 1014.064 & 766.2894 \\
\hline 547.9 & 4093.113 & 2633.955 & 1959.2 & 1548.322 & 1239.863 & 1010.928 & 763.9192 \\
\hline 545.2 & 4072.942 & 2620.975 & 1949.545 & 1540.692 & 1233.753 & 1005.946 & 760.1547 \\
\hline 538.6 & 4023.637 & 2589.246 & 1925.944 & 1522.041 & 1218.817 & 993.7684 & 750.9525 \\
\hline 525.9 & 3928.761 & 2528.193 & 1880.531 & 1486.152 & 1190.078 & 970.3357 & 733.2453 \\
\hline 509.4 & 3805.497 & 2448.871 & 1821.53 & 1439.524 & 1152.74 & 939.8916 & 710.2399 \\
\hline 489.4 & 3656.086 & 2352.724 & 1750.013 & 1383.006 & 1107.481 & 902.9897 & 682.3545 \\
\hline 465.9 & 3480.528 & 2239.751 & 1665.981 & 1316.597 & 1054.302 & 859.6299 & 649.5892 \\
\hline
\end{tabular}


Table 3. (rpm vs. Vehicle Speed per Gear (ft/s))

\begin{tabular}{|c|c|c|c|c|c|c|c|}
\hline rpm & 1 & 2 & 3 & 4 & 5 & 6 & \\
\hline 1000 & 14.01767 & 21.78318 & 29.28538 & 37.05683 & 46.27602 & 56.75569 & 75.1073 \\
\hline 1100 & 15.41944 & 23.9615 & 32.21392 & 40.76251 & 50.90362 & 62.43126 & 82.61803 \\
\hline 1200 & 16.8212 & 26.13981 & 35.14245 & 44.4682 & 55.53123 & 68.10683 & 90.12876 \\
\hline 1300 & 18.22297 & 28.31813 & 38.07099 & 48.17388 & 60.15883 & 73.7824 & 97.63949 \\
\hline 1400 & 19.62474 & 30.49645 & 40.99953 & 51.87956 & 64.78643 & 79.45797 & 105.1502 \\
\hline 1500 & 21.02651 & 32.67477 & 43.92807 & 55.58524 & 69.41403 & 85.13354 & 112.6609 \\
\hline 1600 & 22.42827 & 34.85308 & 46.85661 & 59.29093 & 74.04163 & 90.80911 & 120.1717 \\
\hline 1700 & 23.83004 & 37.0314 & 49.78514 & 62.99661 & 78.66924 & 96.48468 & 127.6824 \\
\hline 1800 & 25.23181 & 39.20972 & 52.71368 & 66.70229 & 83.29684 & 102.1602 & 135.1931 \\
\hline 1900 & 26.63357 & 41.38804 & 55.64222 & 70.40798 & 87.92444 & 107.8358 & 142.7039 \\
\hline 2000 & 28.03534 & 43.56636 & 58.57076 & 74.11366 & 92.55204 & 113.5114 & 150.2146 \\
\hline 2100 & 29.43711 & 45.74467 & 61.4993 & 77.81934 & 97.17965 & 119.187 & 157.7253 \\
\hline 2200 & 30.83887 & 47.92299 & 64.42783 & 81.52503 & 101.8072 & 124.8625 & 165.2361 \\
\hline 2300 & 32.24064 & 50.10131 & 67.35637 & 85.23071 & 106.4348 & 130.5381 & 172.7468 \\
\hline 2400 & 33.64241 & 52.27963 & 70.28491 & 88.93639 & 111.0625 & 136.2137 & 180.2575 \\
\hline 2500 & 35.04418 & 54.45794 & 73.21345 & 92.64207 & 115.6901 & 141.8892 & 187.7682 \\
\hline 2600 & 36.44594 & 56.63626 & 76.14199 & 96.34776 & 120.3177 & 147.5648 & 195.279 \\
\hline 2700 & 37.84771 & 58.81458 & 79.07052 & 100.0534 & 124.9453 & 153.2404 & 202.7897 \\
\hline 2800 & 39.24948 & 60.9929 & 81.99906 & 103.7591 & 129.5729 & 158.9159 & 210.3004 \\
\hline 2900 & 40.65124 & 63.17122 & 34.9276 & 107. & 13 & 915 & 112 \\
\hline 3000 & 42.05301 & 65.34953 & 87.85614 & 111.1705 & 138 & 170.2671 & 3219 \\
\hline 3100 & 43.45478 & 67.52785 & 90.784 & 114.8762 & 4557 & 175. & 3326 \\
\hline 3200 & 44.85655 & 69.70617 & 93.71321 & 118.5819 & 148.0833 & 181.6182 & 240.3434 \\
\hline 3300 & 46.25831 & 71.88449 & 96.6 & 122.2875 & 152 & 187 & \\
\hline 3400 & 47.66008 & 4.0628 & 99.57029 & 125.9932 & 3385 & 192.9694 & 648 \\
\hline 3500 & 49.06185 & 76.24112 & 102.4988 & 129.6989 & 161.9661 & 198.6449 & 262.8755 \\
\hline 3600 & 50.46361 & 78.41944 & 105.4274 & 133.4046 & 166.5937 & 204.3205 & 270.3863 \\
\hline 3700 & 51.86538 & 80.59776 & 108. & 137.1103 & 171.2213 & 209.9 & 897 \\
\hline 3800 & 53.26715 & 82.77607 & 111.2844 & 140.816 & 175.8489 & 215.6716 & 285.4077 \\
\hline 3900 & 54.66891 & 84.95439 & 114.213 & 144.5216 & 180.4765 & 221.3472 & 292.9185 \\
\hline 4000 & 56.07068 & 87.13271 & 117.1415 & 148.2273 & 185.1041 & $227 . C$ & 300.4292 \\
\hline 4100 & 57.47245 & 89.31103 & 120.0701 & 151.933 & 189.7317 & 232.6983 & 307.9 \\
\hline 4200 & 58.87422 & 91.48935 & 122.9986 & 155.6387 & 194.3593 & 238.3739 & 506 \\
\hline 4300 & 60.27598 & 93.66766 & 125.9271 & 159.3444 & 198.9869 & 244.0495 & 322.9614 \\
\hline 400 & 61.67775 & 95.84 & 128 & 163. & 203 & & 0.4721 \\
\hline 4500 & 63.07952 & 98.0243 & 131.7842 & 166.7557 & 208.2421 & 255.4006 & 337.9828 \\
\hline 4600 & 64.48128 & 100.2026 & 134.7127 & 170.4614 & 212.8697 & 261.0762 & 1936 \\
\hline 4700 & 65.88305 & 102.3 & 137.6413 & 174. & 4973 & 266. & 353.0043 \\
\hline 4800 & 67.28482 & 104. & 140.56 & 177.8 & 222 & & 515 \\
\hline 4900 & 68.68658 & 106.7376 & 143.4984 & 181.5785 & 226.7525 & 029 & 368.0 \\
\hline 5000 & 70.08835 & 108.9159 & 146.4269 & 185.2841 & 231.3801 & 283.7785 & 375.5365 \\
\hline 5100 & 71.49012 & $111.0 \mathrm{~s}$ & 149.35 & 188.9 & & 54 & \\
\hline 5200 & 72.89189 & 113.2725 & 152.284 & 192.6955 & 240.6353 & 295.1296 & 390.5579 \\
\hline 5300 & 74.29365 & 115.4508 & 155.2125 & 196.4012 & 245.2629 & 300.8052 & 687 \\
\hline 5400 & 75.69542 & 117.6 & & 200. & 249 & 306 & 794 \\
\hline 5500 & 77.09719 & 119.80 & 161.06 & 203.8126 & 254.5181 & 312.1563 & 413.0901 \\
\hline 5600 & 78.49895 & 121.9858 & 163.9981 & 207.5182 & 259.1457 & 317.8319 & 420.6009 \\
\hline 5700 & 79.90072 & 124.1641 & 166.9267 & 211.2239 & 263.7733 & 323.5074 & 428.1116 \\
\hline 5800 & 81.30249 & 126.3424 & 169.8552 & 214.9296 & 268.4009 & 329.183 & 435.6223 \\
\hline 5900 & 82.70426 & 128.5207 & 172.7837 & 218.6353 & 273.0285 & 334.8586 & 443.1331 \\
\hline 6000 & 84.10602 & 130.6991 & 175.7123 & 222.341 & 277.6561 & 340.5342 & 450.6438 \\
\hline 6100 & 85.50779 & 132.8774 & 178.6408 & 226.0467 & 282.2837 & 346.2097 & 458.1545 \\
\hline 6200 & 86.90956 & 135.0557 & 181.5693 & 229.7523 & 286.9113 & 351.8853 & 465.6652 \\
\hline 6300 & 88.31132 & 37.234 & 184.4979 & 233.458 & 291.5389 & 357.5609 & 473.176 \\
\hline 6400 & 89.71309 & 139.4123 & 187.4264 & 237.1637 & 296.1665 & 363.2364 & 480.6867 \\
\hline 6500 & 91.11486 & 141.5907 & 190.355 & 240.8694 & 300.7941 & 368.912 & 488.1974 \\
\hline 6600 & & & & & & & 082 \\
\hline 6700 & 93.91839 & 145.9473 & 196.212 & 248.2808 & 310.0493 & 380.2631 & 503.2189 \\
\hline
\end{tabular}


M cTighe: Elapsed Time of V ehicle Acceleration

Table 4. (Torque vs. Force Inverse $\left(\mathrm{s}^{2} / \mathrm{ft}\right)$ per Gear)

\begin{tabular}{|c|c|c|c|c|c|c|c|}
\hline torque (lb-ft) & 1 & 2 & 3 & 4 & 5 & 6 & 7 \\
\hline 245 & 0.053788 & 0.083585 & 0.112372 & 0.142193 & 0.177568 & 0.21778 & 0.288198 \\
\hline 278.4 & 0.047335 & 0.073558 & 0.098891 & 0.125134 & 0.156265 & 0.191653 & 0.253623 \\
\hline 306.2 & 0.043037 & 0.066879 & 0.089913 & 0.113773 & 0.142078 & 0.174253 & 0.230596 \\
\hline 329.8 & 0.039958 & 0.062093 & 0.083479 & 0.105631 & 0.131911 & 0.161783 & 0.214095 \\
\hline 349.9 & 0.037662 & 0.058526 & 0.078683 & 0.099563 & 0.124333 & 0.15249 & 0.201796 \\
\hline 367.5 & 0.035859 & 0.055724 & 0.074915 & 0.094795 & 0.118379 & 0.145187 & 0.192132 \\
\hline 382.7 & 0.034434 & 0.05351 & 0.07194 & 0.09103 & 0.113677 & 0.13942 & 0.184501 \\
\hline 396.3 & 0.033253 & 0.051674 & 0.069471 & 0.087906 & 0.109776 & 0.134636 & 0.178169 \\
\hline 408.3 & 0.032275 & 0.050155 & 0.067429 & 0.085323 & 0.10655 & 0.130679 & 0.172933 \\
\hline 419 & 0.031451 & 0.048875 & 0.065707 & 0.083144 & 0.103829 & 0.127342 & 0.168517 \\
\hline 428.7 & 0.03074 & 0.047769 & 0.06422 & 0.081262 & 0.101479 & 0.12446 & 0.164704 \\
\hline 437.5 & 0.030121 & 0.046808 & 0.062929 & 0.079628 & 0.099438 & 0.121957 & 0.161391 \\
\hline 445.4 & 0.029587 & 0.045978 & 0.061812 & 0.078216 & 0.097674 & 0.119794 & 0.158528 \\
\hline 452.7 & 0.02911 & 0.045236 & 0.060816 & 0.076954 & 0.096099 & 0.117862 & 0.155972 \\
\hline 459.3 & 0.028692 & 0.044586 & 0.059942 & 0.075848 & 0.094718 & 0.116168 & 0.153731 \\
\hline 465.4 & 0.028316 & 0.044002 & 0.059156 & 0.074854 & 0.093477 & 0.114646 & 0.151716 \\
\hline 471.1 & 0.027973 & 0.043469 & 0.05844 & 0.073949 & 0.092346 & 0.113259 & 0.14988 \\
\hline 476.3 & 0.027668 & 0.042995 & 0.057802 & 0.073141 & 0.091338 & 0.112022 & 0.148244 \\
\hline 481.2 & 0.027386 & 0.042557 & 0.057214 & 0.072397 & 0.090408 & 0.110881 & 0.146734 \\
\hline 485.7 & 0.027132 & 0.042163 & 0.056684 & 0.071726 & 0.08957 & 0.109854 & 0.145375 \\
\hline 490 & 0.026894 & 0.041793 & 0.056186 & 0.071096 & 0.088784 & 0.10889 & 0.144099 \\
\hline 493.9 & 0.026682 & 0.041463 & 0.055743 & 0.070535 & 0.088083 & 0.10803 & 0.142961 \\
\hline 497.6 & 0.026483 & 0.041154 & 0.055328 & 0.07001 & 0.087428 & 0.107227 & 0.141898 \\
\hline 501.1 & 0.026298 & 0.040867 & 0.054942 & 0.069521 & 0.086817 & 0.106478 & 0.140907 \\
\hline 504.4 & 0.026126 & 0.0406 & 0.054582 & 0.069067 & 0.086249 & 0.105781 & 0.139985 \\
\hline 507.4 & 0.025972 & 0.04036 & 0.054259 & 0.068658 & 0.085739 & 0.105156 & 0.139158 \\
\hline 510.3 & 0.025824 & 0.04013 & 0.053951 & 0.068268 & 0.085252 & 0.104558 & 0.138367 \\
\hline 513.1 & 0.025683 & 0.039911 & 0.053657 & 0.067896 & 0.084787 & 0.103988 & 0.137612 \\
\hline 515.7 & 0.025554 & 0.03971 & 0.053386 & 0.067553 & 0.08436 & 0.103464 & 0.136918 \\
\hline 518.2 & 0.02543 & 0.039518 & 0.053129 & 0.067227 & 0.083953 & 0.102964 & 0.136257 \\
\hline 520.6 & 0.025313 & 0.039336 & 0.052884 & 0.066917 & 0.083565 & 0.10249 & 0.135629 \\
\hline 522.8 & 0.025207 & 0.039171 & 0.052661 & 0.066636 & 0.083214 & 0.102058 & 0.135058 \\
\hline 524.9 & 0.025106 & 0.039014 & 0.05245 & 0.066369 & 0.082881 & 0.10165 & 0.134518 \\
\hline 527 & 0.025006 & 0.038858 & 0.052241 & 0.066105 & 0.082551 & 0.101245 & 0.133982 \\
\hline 528.9 & 0.024916 & 0.038719 & 0.052054 & 0.065867 & 0.082254 & 0.100881 & 0.133501 \\
\hline 530.8 & 0.024827 & 0.03858 & 0.051867 & 0.065632 & 0.08196 & 0.10052 & 0.133023 \\
\hline 532.5 & 0.024748 & 0.038457 & 0.051702 & 0.065422 & 0.081698 & 0.100199 & 0.132598 \\
\hline 534.2 & 0.024669 & 0.038335 & 0.051537 & 0.065214 & 0.081438 & 0.09988 & 0.132176 \\
\hline 535.8 & 0.024595 & 0.03822 & 0.051383 & 0.065019 & 0.081195 & 0.099582 & 0.131782 \\
\hline 537.5 & 0.024517 & 0.038099 & 0.051221 & 0.064813 & 0.080938 & 0.099267 & 0.131365 \\
\hline 538.9 & 0.024454 & 0.038 & 0.051088 & 0.064645 & 0.080728 & 0.099009 & 0.131023 \\
\hline 540.4 & 0.024386 & 0.037895 & 0.050946 & 0.064466 & 0.080504 & 0.098735 & 0.13066 \\
\hline 541.7 & 0.024327 & 0.037804 & 0.050824 & 0.064311 & 0.08031 & 0.098498 & 0.130346 \\
\hline 543.1 & 0.024264 & 0.037707 & 0.050693 & 0.064145 & 0.080103 & 0.098244 & 0.13001 \\
\hline 544.4 & 0.024207 & 0.037616 & 0.050572 & 0.063992 & 0.079912 & 0.098009 & 0.1297 \\
\hline 545.6 & 0.024153 & 0.037534 & 0.050461 & 0.063851 & 0.079736 & 0.097794 & 0.129414 \\
\hline 546.8 & 0.0241 & 0.037451 & 0.05035 & 0.063711 & 0.079561 & 0.097579 & 0.12913 \\
\hline 547.9 & 0.024052 & 0.037376 & 0.050249 & 0.063583 & 0.079402 & 0.097383 & 0.128871 \\
\hline 549 & 0.024004 & 0.037301 & 0.050148 & 0.063456 & 0.079243 & 0.097188 & 0.128613 \\
\hline 550.1 & 0.023956 & 0.037227 & 0.050048 & 0.063329 & 0.079084 & 0.096994 & 0.128356 \\
\hline 549.6 & 0.023978 & 0.037261 & 0.050093 & 0.063386 & 0.079156 & 0.097082 & 0.128473 \\
\hline 547.9 & 0.024052 & 0.037376 & 0.050249 & 0.063583 & 0.079402 & 0.097383 & 0.128871 \\
\hline 545.2 & 0.024171 & 0.037561 & 0.050498 & 0.063898 & 0.079795 & 0.097865 & 0.129509 \\
\hline 538.6 & 0.024467 & 0.038022 & 0.051116 & 0.064681 & 0.080773 & 0.099065 & 0.131096 \\
\hline 525.9 & 0.025058 & 0.03894 & 0.052351 & 0.066243 & 0.082723 & 0.101457 & 0.134262 \\
\hline 509.4 & 0.02587 & 0.040201 & 0.054046 & 0.068389 & 0.085403 & 0.104743 & 0.138611 \\
\hline 489.4 & 0.026927 & 0.041844 & 0.056255 & 0.071184 & 0.088893 & 0.109024 & 0.144276 \\
\hline 465.9 & 0.028285 & 0.043955 & 0.059093 & 0.074774 & 0.093377 & 0.114523 & 0.151553 \\
\hline
\end{tabular}


Table 5. (Transmission Specifications and Tire Radius)

\begin{tabular}{|c|c|c|c|}
\hline Differential Ratio & Gear & Ratio & Tire Radius (ft) \\
\hline 3.666 & 1 & 3.397 & 1.667 \\
\hline & 2 & 2.186 & \\
\hline & 3 & 1.626 & \\
\hline & 4 & 1.285 & \\
\hline & 5 & 1.029 & \\
\hline & 6 & 0.839 & \\
\hline & 7 & 0.634 & \\
\hline
\end{tabular}

